\title{
Diagnosis of Ankylosing Spondylitis: Do not let the spine bites the eye
}

\author{
Chia Chee Chew, Ju Juen Chin, Wan Hazabbah Wan Hitam, Mei Fong Chong, \\ Liza-Sharmini Ahmad Tajudin
}

Chew CC, Chin JJ, Wan Hitam WH, et al. Diagnosis of Ankylosing Spondylitis: Do not let the spine bites the eye . Malays Fam Physician.

2021;16(2);73-77. https://doi.org/10.51866/cr1121

\section{Keywords:}

Low back pain, ankylosing

spondylitis, anterior uveitis

good health and well being

\section{Authors:}

\begin{abstract}
Liza-Sharmini Ahmad Tajudin
(Corresponding author)

MBBS, MMed, PhD, AM

Department of Ophthalmology and Visual Science, School of Medical

Sciences, UniversitiSains Malaysia

Kelantan, Malaysia
\end{abstract}

Ophthalmology clinic, Hospital USM UniversitiSains Malaysia, Kelantan Malaysia

\section{Chew Chia Chee}

$M D$

Department of Ophthalmology and Visual Science, School of Medical Sciences, UniversitiSains Malaysia Kelantan, Malaysia

Ophthalmology clinic, Hospital USM UniversitiSains Malaysia, Kelantan Malaysia

Ophthalmology clinic, Hospital Raja PermaisuriBainun, Ipoh, Perak Malaysia

\begin{abstract}
Background: A diagnosis of ankylosing spondylitis (AS) is challenging and often delayed despite patients being symptomatic. Low back pain is the most common initial symptom, appearing in the second and third decades of life. Acute anterior uveitis (AAU) occurs much later in the course of the disease, often when the destruction of the spine is already debilitating.

Objective: Here, we report three cases of AS that were diagnosed after the patients developed AAU. Methods: A case series illustrated AAU leading to the diagnosis of AS years after the initial episode of low back pain. A comparison of the clinical presentation, diagnosis, and outcomes was also illustrated.

Result: We report three cases of acute anterior uveitis (AAU)-associated AS diagnosed only after many visits to the primary health care provider with the complaint of chronic low back pain. All three patients had irreversible radiological changes upon diagnosis of AS. The AAU resolved with topical steroids, and one patient developed cataract.

Conclusion: A high index of suspicion of AS in a young adult with chronic back pain before the development of AAU may prevent further functional loss and provide a better prognosis. Diagnosis of AS following AAU is not only associated with dependency but also may rob the vision of a young adult.
\end{abstract}

\section{Introduction}

Low back pain is a frequent symptom that brings the patient to primary healthcare. The pain is defined as chronic when it lasts for more than 12 weeks. Low back pain is among the earliest symptoms of ankylosing spondylitis (AS). AS imposes a significant economic burden globally due to a reduction in productivity. ${ }^{1}$ It predominantly affects males more than females (3:1 ratio) and peaks during reproductive age. ${ }^{2}$ Sacroiliitis and inflammation of the spine are the most common features in AS. Forty-five percent of patients present with acute anterior uveitis (AAU), which is the most common extraarticular manifestation during the disease. ${ }^{3}$ This report aims to create awareness among primary care practitioners to have a high index of suspicion for AS, especially in young male patients in their early twenties with chronic low back pain before they develop AAU, which only happens late in the disease.

\section{Case Report}

\section{Case 1}

A 53-year-old man was referred to an eye clinic from a primary care clinic after experiencing right eye pain and redness with reduced vision for a week. He had a similar episode of painful red eye previously and was treated by a primary care practitioner. His vision improved after treatment. He also had chronic back pain for the past 20 years, starting when he was in his early thirties, which had been treated with analgesics in different primary care centres.

The visual acuity (VA) of the patient's right eye (OD) was 6/18 and was 6/24 in the left eye (OS). There was conjunctival hyperaemia with severe anterior chamber inflammation (cells of $4+)$, fibrin clumps, and posterior synechiae in the OD (Figure 1A). There were also seclusio pupillae and visually significant cataract OS but without cellular activity (Figure 1B). B-scan imaging showed the presence of vitritis OD. Schober's test was positive with only an increment of $1 \mathrm{~cm}$ upon lumbar flexion. The Fleche test was also positive, indicating the involvement of the cervical spine. Erythrocyte sedimentation rate (ESR) was raised (43 millimetres/hour), and HLA-B27 was positive. There was the presence of syndesmophytes in the lumbosacral and cervical radiograph, which 


\section{Chin Ju Juen}

Department of Ophthalmology and Visual Science, School of Medical Sciences, UniversitiSains Malaysia Kelantan, Malaysia

Ophthalmology clinic, Hospital USM UniversitiSains Malaysia, Kelantan Malaysia

Ophthalmology clinic, Hospital Raja PermaisuriBainun, Ipoh, Perak Malaysia

\section{Wan Hazabbah Wan Hitam}

Department of Ophthalmology and Visual Science, School of Medical Sciences, UniversitiSains Malaysia Kelantan, Malaysia

Ophthalmology clinic, Hospital USM UniversitiSains Malaysia, Kelantan Malaysia

\section{Chong Mei Fong}

Ophthalmology clinic, Hospital Raja PermaisuriBainun, Ipoh, Perak Malaysia gave rise to the classical "bamboo spine" appearance (Figure 2A\& 2B). He was diagnosed with severe anterior uveitis OD and quiescent anterior uveitis OS. He was treated with a tapering dose of topical steroids for 3 months. VA of the OD improved to $6 / 6$ but remained the same in the OS due to cataract. Diagnosis of AS was made by the rheumatologist. The patient was treated with a non-steroidal anti-inflammatory drug (NSAID) and sulfasalazine.

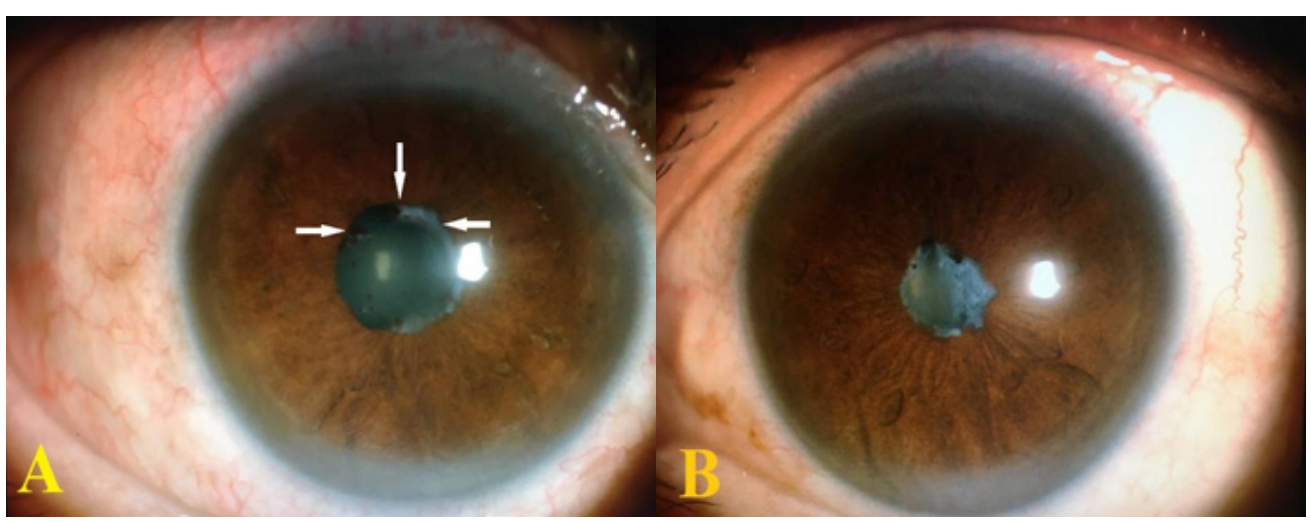

Figure 1A: Slit lamp image of the OD showing conjunctival hyperaemia, posterior synechiae (arrow), and irregular pupil. Figure 1B: Slit lamp image of the OS showing seclusio pupillae secondary to extensive posterior synechiae and the presence of cataract as a complication of AAU.

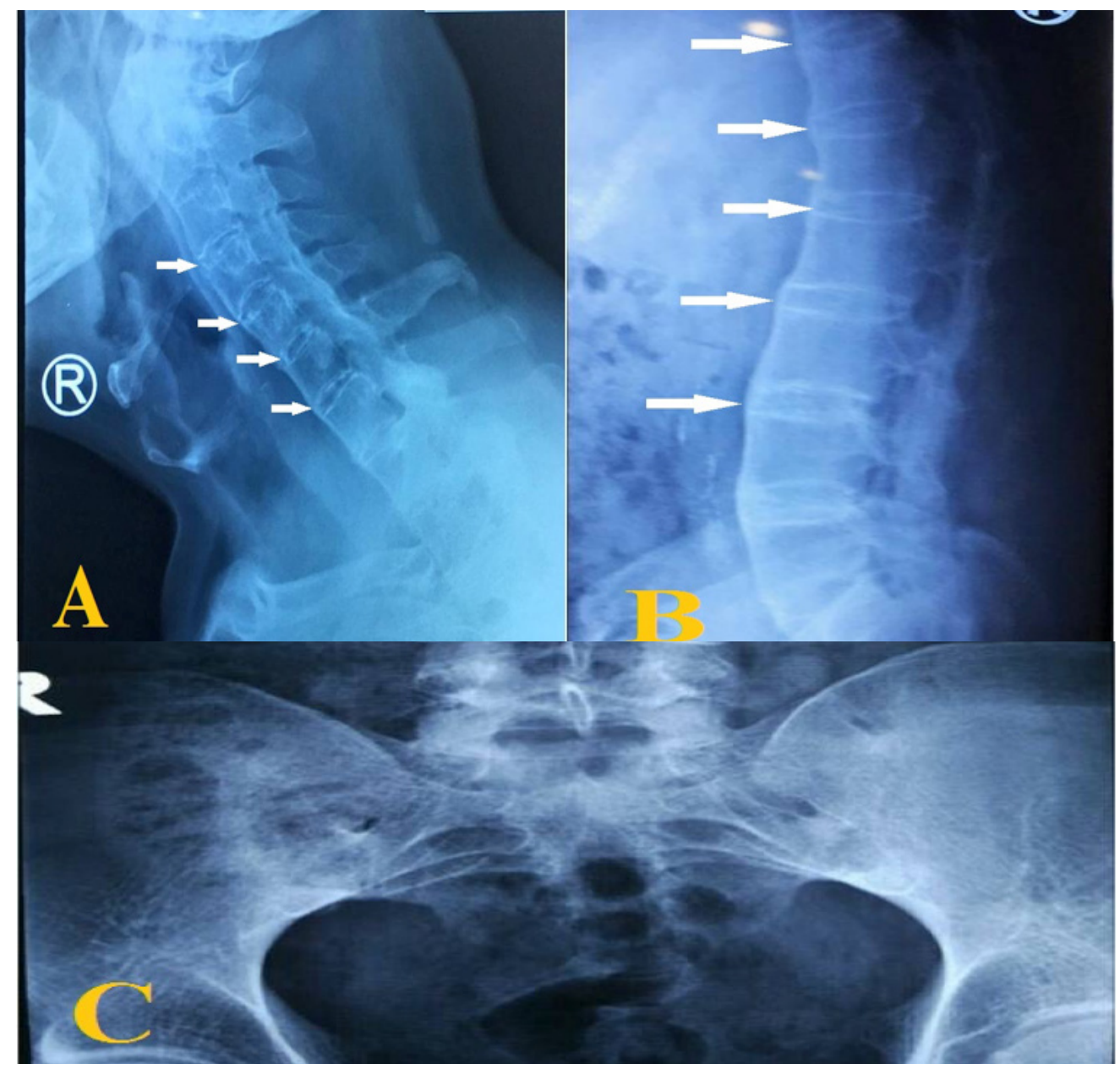

Figure 2A: Cervical radiograph showing the presence of syndesmophytes and loss of cervical lordosis.

Figure 2B: Lateral lumbar radiograph showing the presence of syndesmophytes, giving rise to the classical "bamboo spine" appearance.

Figure 2C: Pelvic radiograph (anteroposterior view) showing bilateral fusion of sacroiliac joints (SIJ). 


\section{Case 2}

A 56-year-old man was referred from a primary care practitioner with a recurrent painful red right eye for two weeks; the last attack of a painful right red eye had been 10 years ago and was self-treated with over-thecounter medications. For the previous 15 years, he had a history of chronic low back pain that required analgesia occasionally. On examination, his VA was $6 / 36 \mathrm{OD}$ and $6 / 6$ OS. Slit lamp examination of his OD showed circum-corneal injection with intense anterior chamber reaction $(4+$ cells), fibrin, and keratic precipitates at the inferior cornea. There was also posterior synechiae from 1 to 10 o'clock (Figure 3). The OS was unremarkable. Posterior segment examination of both eyes was normal. Schober's test was positive with a $3 \mathrm{~cm}$ increment on lumbar flexion. Other systemic examinations were unremarkable. His ESR was raised at 77 millimetres/hour, and HLA-B27 was positive. A lumbosacral radiograph showed irregular widening of the right SIJ and sclerosis of the left SIJ with bilateral grade 3 sacroiliitis (Figure 4). He was treated as a case of severe anterior uveitis OD, and a topical steroid was prescribed. He was subsequently reviewed by the rheumatologist, diagnosed with AS, and given oral NSAIDs. His vision recovered to $6 / 6$, and anterior chamber inflammation of the OD resolved.

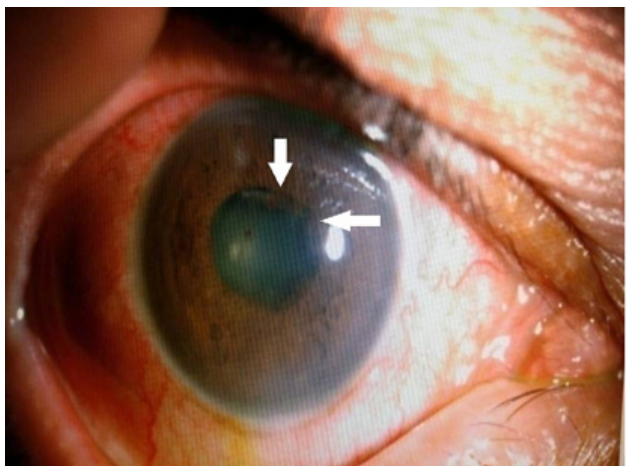

Figure 3: Anterior segment photograph of the OD showing conjunctival hyperaemia and posterior synechieae (arrows)

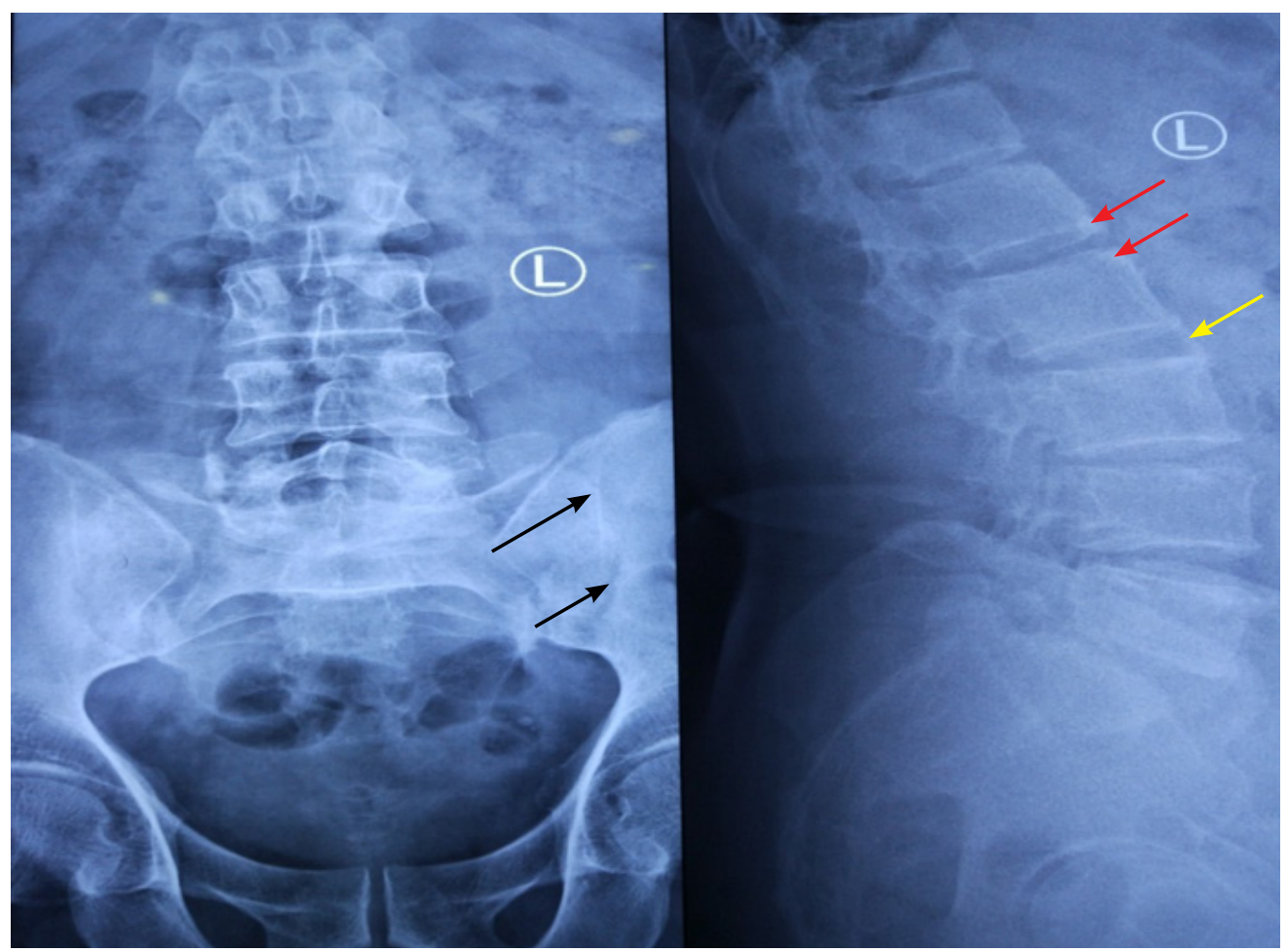

Figure 4: Lumbosacral radiograph of the SIJ showing irregular widening of the joint space on the right SIJ (white arrowhead), erosion, and decreased joint space (arrow) over the left SIJ. Lateral view of lumbar radiograph showing syndesmophyte (yellow arrow) and sclerosis at the superior and inferior endplates of the vertebral bodies and corner sign (red arrow).

\section{Case 3}

A 31-year-old man presented with redness, foreign body sensation, and mild reduction of vision of OS for 5 days. He had intermittent backache for the previous 5 years with multiple visits to different primary care practitioners. He was treated symptomatically with analgesia. On examination, the VA was 6/9 for both eyes. Anterior segment examination of the OS showed conjunctival hyperaemia with intense anterior chamber reaction (4+ cells and fibrin clumps) and the presence of keratic 
precipitates. The conjunctiva was white on OD, but there was mild anterior chamber reaction with cells of $2+$. Schober's test was positive with an increment of $3 \mathrm{~cm}$, but the Fleche test was normal. His ESR was raised at 58 millimetres/hr. He was treated with topical steroids for bilateral eyes anterior uveitis. A lumbosacral radiograph showed fusion of both SIJ (Figure 5). A diagnosis of AS was made by the rheumatologist, and he was treated with NSAIDs.

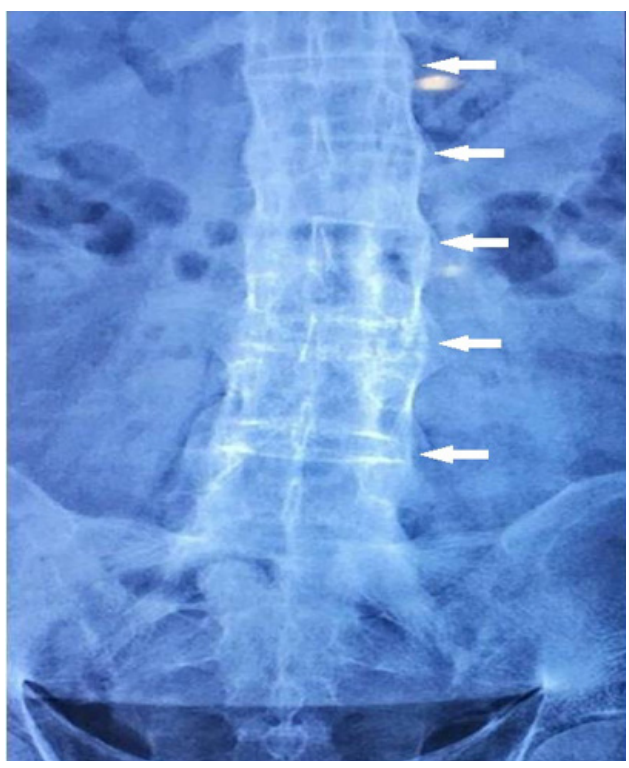

Figure 5: Lumbosacral radiograph showing the presence of syndesmophytes (arrow) and fusion of bilateral SIJ.

\section{Discussion}

A 7- to 10-year diagnostic delay of AS is commonly seen up due to the insidious onset of inflammatory low back pain before demonstrating characteristic radiographic changes. ${ }^{2}$ Diagnosis of AS was delayed in all cases in this series. Case 1 and case 2 were only diagnosed after a 20- and a 15-year history of low back pain, respectively. The patients had already suffered from a loss of spinal motility and function when the diagnosis was made. In Malaysia, back pain is rated as the ninth and fifth most common complaint in public and private health care clinics, respectively. ${ }^{4}$ An estimated prevalence of low back pain secondary to AS in primary care was only $0.3 \%{ }^{5}$; nevertheless, it is crucial to ensure an accurate diagnosis and prompt treatment of this debilitating disease.
Plain radiography or computed tomography may not be as sensitive as MRI to detect sacroiliitis at the early course of AS. ${ }^{2}$ However, MRI is expensive and not recommended as a routine investigation. Assessment of Spondyloarthritis International Society for Inflammatory Back Pain (ASAS IBP) is a useful screening tool to detect inflammatory back pain. ${ }^{6}$ ASAS IBP proposed the mnemonic of iPAIN (based on the symptoms): Insidious onset; Pain at night; Age at onset less than 40 years old; Improvement with exercise; No improvement with rest. The sensitivity is reported to be $77 \%$ and the specificity $91.7 \%$ if 4 out of 5 criteria are fulfilled. ${ }^{6}$ Primary care practitioners should be aware of the iPAIN for prompt referral to a rheumatologist, early diagnosis, and appropriate management to prevent disease complications.

AAU with HLA-B27 expression is reported to be associated with an increased risk of developing AS. ${ }^{7}$ HLA-B27 was positive in all cases in this case series. HLA-B27 is recommended to be included in the list of investigations for AAU especially in those with chronic low back pain. The visual outcome of AAU is good, although recurrence is inevitable in many cases. Complications of AAU, such as cataracts and secondary glaucoma, are common and unavoidable. However, the structural damages of the spine were unchangeable even when inflammation of the eyes was under control.

Early treatment of AS with NSAIDs provides modest improvement in terms of pain, function, and disease activity. ${ }^{8}$ Long-term treatment with NSAIDs may be effective in delaying progression in certain subgroups of patients. $^{8}$

\section{Conclusion}

AS is a debilitating disease that causes structural and functional loss. Early detection and treatment may reduce disease activity for patients and improve quality of life. Practitioners should not let the signs of AAU guide the diagnosis of AS, because this is waiting for the 'spine to bite the eye'. 


\section{How does this paper make a difference to general practice?}

- This paper highlights the importance of routine care to detect AS before characteristic radiological findings appear. It also raises awareness of AS among primary care practitioners.

- Early diagnosis and treatment of AS are vital to improving symptoms, disease activity, and skeletal function.

- Delayed recognition of this disease can be prevented by having a high index of suspicion for AS among young patients with chronic low back pain.

- Collaboration between tertiary health care practitioners is advisable to expedite the referral of this elusive disease.

\section{References}

1. Ramos-Remus C, Hernandez-Rios G, DuranBarragan S, et al. Fifteen-year trends of long term disability and sick leaves in ankylosing spondylitis. Clin Rheumatol. 2011;30(3):361-7.

2. Shaikh SA. Ankylosing spondylitis: recent breakthroughs in diagnosis and treatment. J Chiropr Assoc. 2007 Dec;51(4):249-60.

3. Bacchiega ABS, Balbi GGM, Ochtrop MLG, et al. Ocular involvement in patients with spondyloarthritis. Rheumatology. 2017 Dec;56 (12):2060-2067
4. Hani SS, Liew SM. The views and experiences of Malaysian primary care doctors in managing patients with chronic low back pain: a qualitative study. Malays Fam Physician. 2018;13(1):18-27.

5. Deyo RA, Weinstein JN. Low back pain. $N$ Engl J Med 2001; 344:363-370.

6. Weisman MH. Inflammatory back pain: the United States perspective. Rheum Dis Clin North Am. 2012 Aug;38(3):501-12.
7. Yen JC, Hsu CA, Hsiao SH, et al. Acute anterior uveitis as risk factor of ankylosing spondylitis-A national population-based study. Int J Environ Res Public Health. 2017;14(1):107.

8. Kroon FP, van der Burg LR, Ramiro S, et al. Non-steroidal anti-inflammatory drugs (NSAIDs) for axial spondyloarthritis (ankylosing spondylitis and non-radiographic axial spondyloarthritis). Cochrane Database Syst Rev. 2015;(7):CD010952. 